\section{P149 DISCUSSING MENTAL HEALTH WITH YOUNG PEOPLE ATTENDING SEXUAL AND REPRODUCTIVE HEALTH SERVICES}

Kimberley Forbes, Elizabeth Okecha*, Richard West. Sexual Health Hounslow, London, UK

10.1136/sextrans-2015-052126.192

Background/introduction Within Sexual and Reproductive Health (SRH) clinics identification of Mental Health $(\mathrm{MH})$ problems is an important part of a consultation with young people (YP).

Aim(s)/objectives To review the number of YP who had documentation of a conversation regarding $\mathrm{MH}$.

Methods Electronic patient records of 103 attendees were selected at random and reviewed.

Results $\mathrm{MH}$ discussion was documented in $81 \%$ (26/32) of $<16$ s, 67\% $(\mathrm{n}=20 / 30)$ aged $16,37 \%(\mathrm{n}=15 / 41)$ of those aged 17-18 years. Of these Child and Adolescent MH Services (CAMHS) were accessed by $23 \%(6 / 26)<16$ s (2/6 lost FU), $15 \%(n=3 / 20)$ aged 16 and $7 \%(n=1 / 15)$ aged $17-18$ years. Of these ten disclosed the following specific disorders ADHD (2), self-harm (3), depression (2), anorexia and past sexual abuse (1) and conduct disorder (1), suicidal thoughts (1). 3/9 aged 16 and under who had accessed CAMHS disclosed sexual abuse.

Discussion/conclusion Sexual health is an important access point for YP with mental health problems, new or lost to follow up and may be associated with a disclosure of sexual abuse. Significant pressures exist in CAMHS services. Shared clinical experience and robust links between sexual health, CAMHS, general practice and youth services with appropriate referral pathways are important. We recommend training for all SRH staff should include: skills in eliciting $\mathrm{MH}$ problems in all consultations with YP, awareness of common $\mathrm{MH}$ problems in adolescence and knowledge of local service configuration including thresholds for referral to appropriate providers.

\section{P150 CREATING OPPORTUNITIES FROM LOCAL AUTHORITY COMMISSIONING: EARLY INTERVENTION PATHWAY IN YOUNG PEOPLE'S SEXUAL HEALTH SERVICES}

Kimberley Forbes, Elizabeth Okecha*, David Daniels, Richard West. Sexual Health Hounslow, London, UK

\subsection{6/sextrans-2015-052126.193}

Background/introduction Young people (YP) seen within sexual health services (SHS) may not meet referral thresholds for traditional social care measures but could benefit from improved links to early intervention services (EIS) such as targeted youth support.

Aim(s)/objectives The aim was to assess needs of attendees to inform service delivery and review use of a local safeguarding assessment proforma and review concerns identified.

Methods We reviewed a random selection of 103 records from attendees 18 or younger attending in 2013 identified by the clinic management system.

Results 18 male (17.5\%). Where documented 24/68 (35\%), $13 / 39$ (33\%) and 34/44 (77\%) reported current smoking, drug and alcohol use respectively. $32<16 \mathrm{~s}$ had a proforma including decision regarding referral to social care within 12 months (100\%), 8 were known to social care (25\%). 5 reported nonconsensual sex (17\%) and 10 reported searched at age 13 or younger (31\%). No infections were diagnosed in $<16$ s. $28 / 30$ and 29/41 of those aged $16(93 \%)$ and $17-18$ years (71\%) respectively had a completed proforma.

Discussion/conclusion YP attending SHS have a number of vulnerabilities that do not meet safeguarding intervention thresholds. We have developed a holistic approach by: developing pathways between SHS and EIS, recruitment of a Relationships Worker to provide targeted support and staff training in understanding and recognising additional needs and vulnerabilities which exist even in the absence of infections.

\section{P151 REATTENDANCE RATES IN MEN PRESENTING WITH SYMPTOMS OF URETHRITIS - SHOULD WE BE DOING BETTER?}

${ }^{1,2}$ Naomi Carter*, 'Megan Crofts, ${ }^{1}$ Michael Clarke, ${ }^{1}$ Helen Wheeler, ${ }^{2}$ Debbie Sharp, 1,2 2 addy Horner. 'Bristol Sexual Health Centre, Bristol, UK; ${ }^{2}$ University of Bristol, Bristol, UK

10.1136/sextrans-2015-052126.194

Background Chlamydia and gonorrhoea are common causes of urethritis. Management is often based on an enhanced syndromic approach while awaiting results. This can necessitate prescribing to cover a range of potential pathogens, and result in uncertainty for patients. Point of care testing (POCT) for chlamydia and gonorrhoea in men with symptoms of urethritis could alter care pathways and reduce reattendance.

Aims To measure reattendance rates in men presenting with symptoms of urethritis. To identify reasons for reattendance including those that could be mitigated by POCT.

Methods All men with urethritis symptoms presenting over a three month period were identified using electronic patient records. Urethritis was defined as $\geq 5 \mathrm{pmnls} / \mathrm{hpf}$ on a Gram stained urethral smear. Reattendances within 30 days of initial clinic visit and reasons for reattendance were recorded for both microscopy-positive and negative groups.

Results 431 men with urethritis symptoms were identified in a 3 month period. 192 had confirmed urethritis on initial microscopy. $31 \%$ of microscopy-positive men and $42 \%$ of microscopynegative men reattended at least once within 30 days of initial visit. Common reasons for reattendance were early morning smear (20\%), persistent symptoms (18\%), results (16\%) and gonorrhoea test of cure (9\%).

Discussion This service evaluation has identified high reattendance rates in men with urethritis symptoms. POCT could impact on reattendance rates in a number of ways. Pathogen-guided treatment may reduce antimicrobial failure and persistent symptoms. Same-day results could reduce results visits. Reassurance from negative same-day results may also have a role in reducing persistent symptoms.

\section{P152 WHAT KIND OF INFORMATION DO PATIENTS WANT TO SEE IN SEXUAL HEALTH CLINIC WAITING ROOMS?}

${ }^{1}$ William Spice*, ${ }^{1}$ Rebecca Clamp, ${ }^{2}$ Claire Palmer. ${ }^{1}$ Worcestershire Health and Care Trust, Worcester, UK; ${ }^{2}$ University Hospital Birmingham, Birmingham, UK

\subsection{6/sextrans-2015-052126.195}

Background/introduction All sexual health clinics have noticeboards and leaflets in their waiting rooms carrying a range of information, but little is known about the kind of information patients find most useful. This survey was designed to gain 